\title{
Caractérisation thermique d'un multijet tourbillonnaire en mode confiné avec et sans obstacle
}

\author{
Mohamed Braikia ${ }^{\text {a }}$, Ali. Khelil ${ }^{\text {a }}$ Larbi Loukarfi ${ }^{a}$ Hassan Naji ${ }^{b}$ \\ ${ }^{a}$ Laboratoire de contrôle, essais, mesures et simulation mécanique, Université Hassiba Benbouali de CHLEF, B.P. \\ 151, 02000 Chlef, Algérie. \\ ${ }^{b}$ Laboratoire Génie Civi \& Géo-Environnement (LGCgE- EA 4515), Université d'Artois/Faculté des Sciences \\ Appliquées, F-62400 Béthune, France.
}

\begin{abstract}
Résumé La compréhension des effets du confinement d'un multi jet tourbillonnaire, sur le taux d'entraînement de l'air et sur la stratification de la température est très importante pour l'efficacité du processus de conditionnement d'air. Les jets impactant sont un moyen efficace d'optimisation des transferts thermiques entre un fluide et une structure solide. Ils sont, à ce titre, utilisés dans diverses applications industrielles telles que les processus de rafraichissement, de réchauffement ou de séchage. Une série d'études expérimentales a été menée pour déterminer l'influence de différents facteurs sur les caractéristiques et la structure du jet tourbillonnaire multiple permettant d'obtenir le mélange permettant l'homogénéisation de l'ambiance dans les locaux et de même dans les chambres de combustion.
\end{abstract}

\section{Introduction}

Une mauvaise diffusion du jet d'air froid dans les locaux, liée à un défaut de mélange du jet froid de climatisation à l'air chaud de cet espace conduit a des sensations d'inconfort pour les occupants, pouvant se traduire par des maux de têtes. Dans le cas des enceintes confinées à fortes charges internes tel un habitacle de transport, le contrôle du jet d'air froid et de son brassage à l'air ambiant est encore plus difficile. La forte charge interne conduit à des conditions extrêmes de soufflage, au détriment des conditions de confort souhaitées.

Les jets confinés ont fait l'objet de plusieurs travaux de recherches expérimentales et numériques. La plupart des travaux effectués sur les jets confinés sont orientés vers les chambres de combustion des moteurs, des réacteurs nucléaires et des brûleurs afin d'obtenir un mélange homogène entre carburant et comburant, mais aussi dans les cas d'études des risques inhérents aux incendies en milieu confiné et le contrôle des mouvements de masses gazeuses (contrôle des écoulements de fumées) dans les incendies en espaces semi-confinés $[1,2,3]$,

Le soufflage par des buses, a diffusion tourbillonnaire, est l'un des meilleurs moyens passifs de mise en œuvre pratique du contrôle de l'écoulement d'air. Ces diffuseurs induisent une amélioration du abraikia_m@yahoo.fr confort thermique, conduisant ainsi à une économie d'énergie [4].

Dans la présente étude, l'interaction de jets tourbillonnaires en mode confiné est étudiée expérimentalement, selon différentes configurations d'écoulements avec et sans obstacle pour caractériser l'interaction entre jets tourbillonnaires, afin d'homogénéiser thermiquement, une ambiance.

L'amélioration de la qualité du mélange permettrait de minimiser la consommation énergétique tout en respectant les contraintes liées au type d'application.

\section{Dispositif expérimental}

Le banc expérimental est une enceinte de dimensions $(1,20 \times 1,20 \times 2,00 \mathrm{~m})$ (Fig.1). Le dispositif de soufflage, comporte des diffuseurs de soufflage d'air chaud dirigés vers le bas. Les températures de l'écoulement sont mesurées par un thermo-anémomètre multifonctionnel. Le système multi-jets étudié est une couronne de jets hélicoïdaux entourant un jet hélicoïdal central. Le débit s'opère à un nombre de Reynolds, de l'ordre de $3.10^{4}$. L'étude a été réalisée dans des conditions de flux de chaleur uniforme pour chaque diffuseur. 


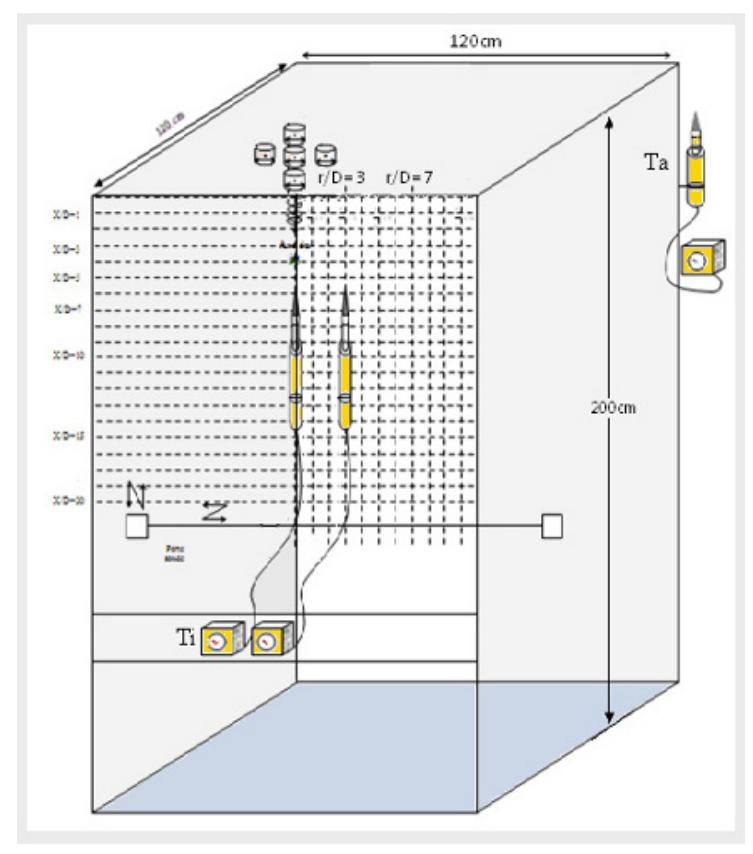

Figure 1. Enceinte expérimentale du multi jet tourbillonnaire.

\section{Système multi-jet tourbillonnaire en mode confiné sans obstacle}

Le système multi jets étudié est constitue d'une couronne de 6 jets tourbillonnaires entourant un jet central tourbillonnant en sens inverse Figure 2.

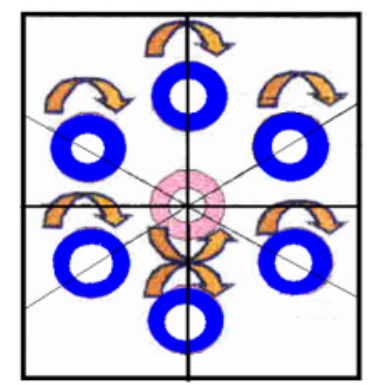

Figure 2. Configuration utilisée.

La compréhension des effets du confinement d'un multi jets tourbillonnement, sur le taux d'entraînement de l'air et sur la stratification de la température est très importante pour l'efficacité du processus de conditionnement d'air. Pour apprécier cet effet, nous présentons sur la figure (3) les profils de températures axiales et radiales d'un multi jets tourbillonnaire en mode confiné.

Les températures axiales et radiales de l'écoulement sont obtenues par référence à la température maximale moyenne à la sortie de l'orifice de soufflage et à la température ambiante grâce à une expression adimensionnelle de la forme :

$$
\operatorname{Tr}=[(T i-T a) /(T \max -T a)]
$$

Les distances radiales et axiales sont données par référence au diamètre de l'orifice de soufflage sous forme adimensionnelle $\mathrm{r} / \mathrm{D}$ et $\mathrm{x} / \mathrm{D}$.
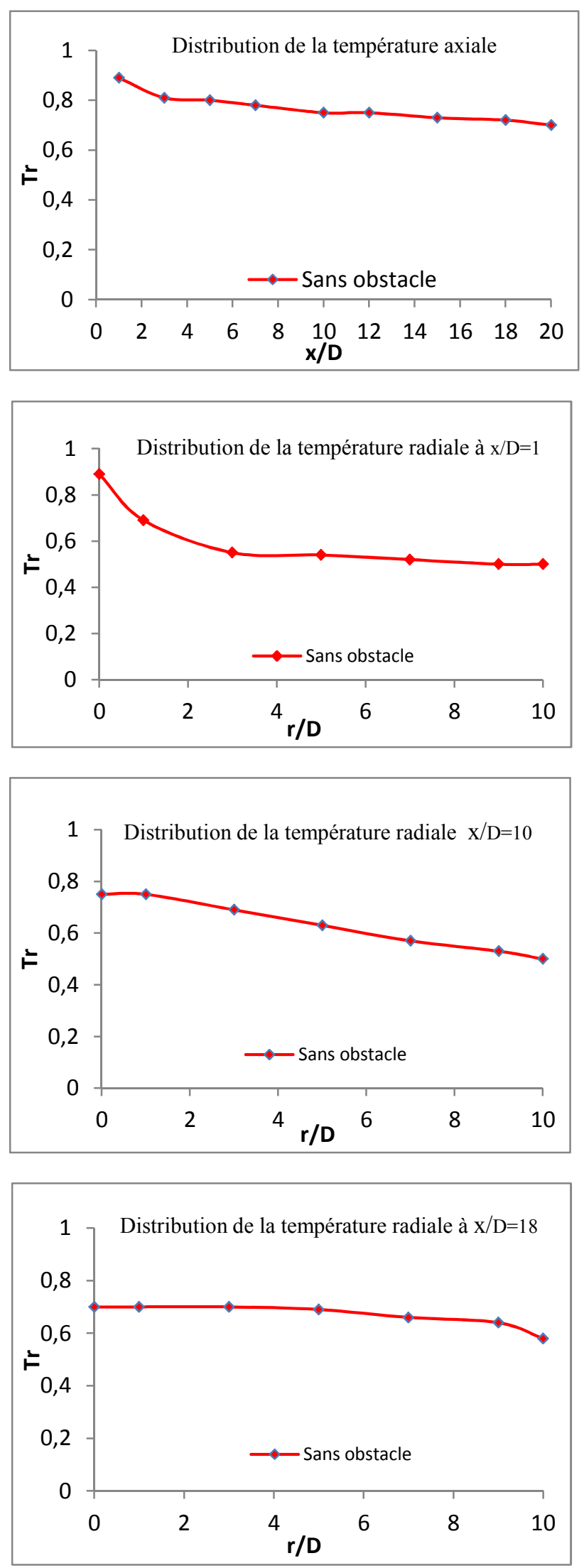

Figure 3. Profils des températures axiales et radiales d'un multi jets tourbillonnaire en mode confiné.

\section{Système multi-jet tourbillonnaire en mode confiné avec obstacle}


Les jets impactant permettent d'optimiser les transferts thermiques entre un fluide et une structure solide. Ils sont, à ce titre, utilisés dans diverses applications industrielles telles que les processus de refroidissement, de réchauffement ou de séchage $[5,6]$.

Les profils de température axiale et radiale du multi jet tourbillonnaire en mode confiné, dans le cas de la présence d'un obstacle placé à différentes positions axiales sont présentés sur les figures $4,5,6$ et 7 .

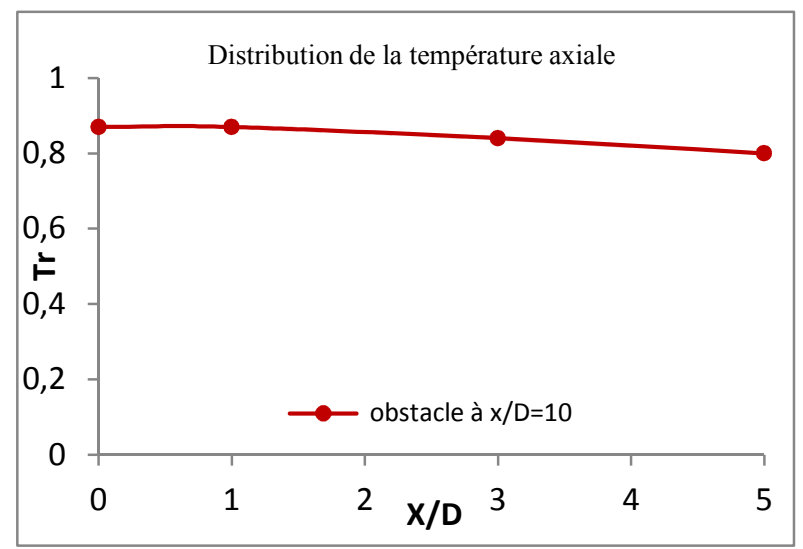

Figure 4. Profil de la température axiale d'un multi jets tourbillonnaire en mode confiné avec obstacle à $\mathrm{x} / \mathrm{D}=10$.

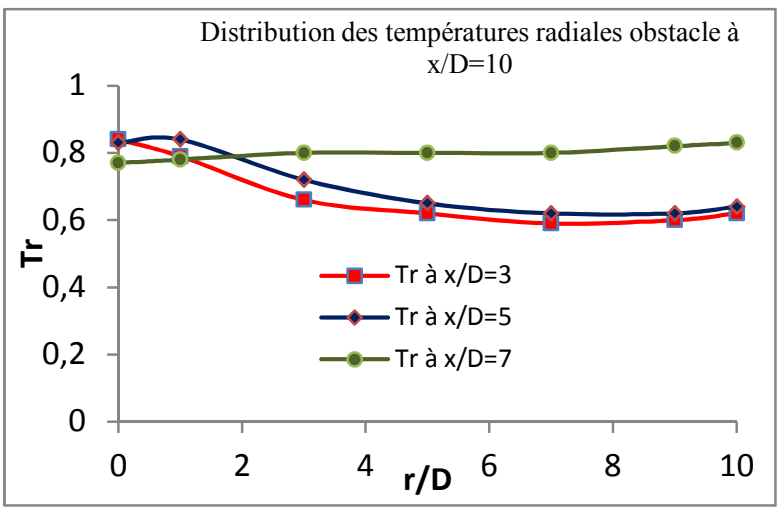

Figure 5. Profils de la température radiale d'un multi jets tourbillonnaire en mode confiné avec obstacle à $\mathrm{x} / \mathrm{D}=10$

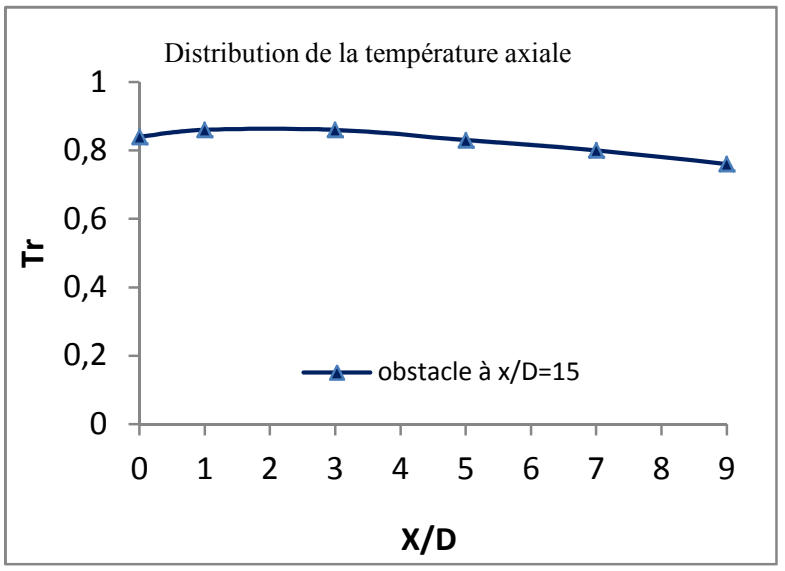

Figure 6. Profil de la température axiale d'un multi jets tourbillonnaire en mode confiné avec obstacle à $\mathrm{x} / \mathrm{D}=15$

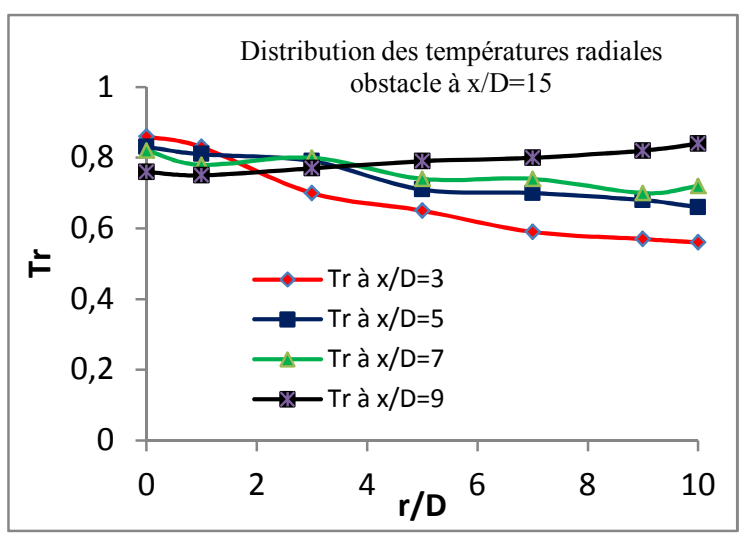

Figure 7. Profils de la température radiale d'un multi jets tourbillonnaire en mode confiné avec obstacle à $\mathrm{x} / \mathrm{D}=15$

L'évolution des profils de températures radiales du multi jets tourbillonnaire en mode confiné avec un obstacle à $\mathrm{x} / \mathrm{D}=10$, puis à $\mathrm{x} / \mathrm{D}=15$, pour les différentes stations axiales étudiées, montre que la présence de l'obstacle assure une meilleure homogénéisation des températures radiales, au fur et mesure que l'on s'éloigne de l'orifice de soufflage, et ce, dans toute la section du jet résultant.

Ainsi dans le cas où l'obstacle est placé à $\mathrm{x} / \mathrm{D}=10$, l'homogénéisation de la température radiale est notable à sept diamètres avec une amplitude plus importante au bord du jet résultant. Cela est dû au retour d'air chaud, provoqué par la présence de l'obstacle et du confinement. Cette constatation est confirmée pour une autre position de l'obstacle ( $\mathrm{x} / \mathrm{D}=15)$.

On constate également, une homogénéisation des températures radiales à faible distance de l'orifice de soufflage, et dans toutes les sections du jet. Ainsi, la présence de l'obstacle permet un étalement appréciable au bord du jet résultant et le confinement en présence de l'obstacle favorise d'une manière non négligeables la stabilité en température et ce, d'autant plus lorsqu'on s'éloigne de l'orifice de soufflage. On remarque aussi que lorsque la distance axiale augmente, le profil de température radiale tend à s'aplatir pour fournir une stabilité thermique et un étalement important.

Sur la figure 8, nous comparons les profils de températures axiales et radiales en mode confiné avec et sans obstacle.

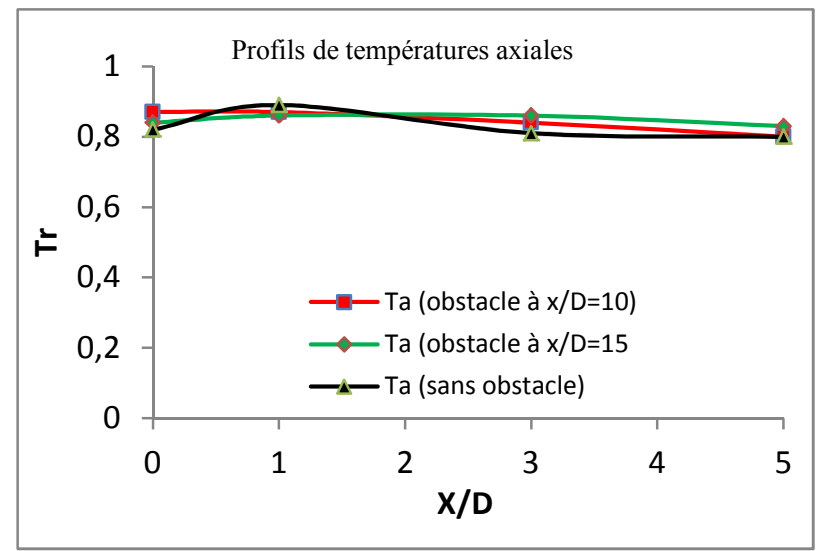



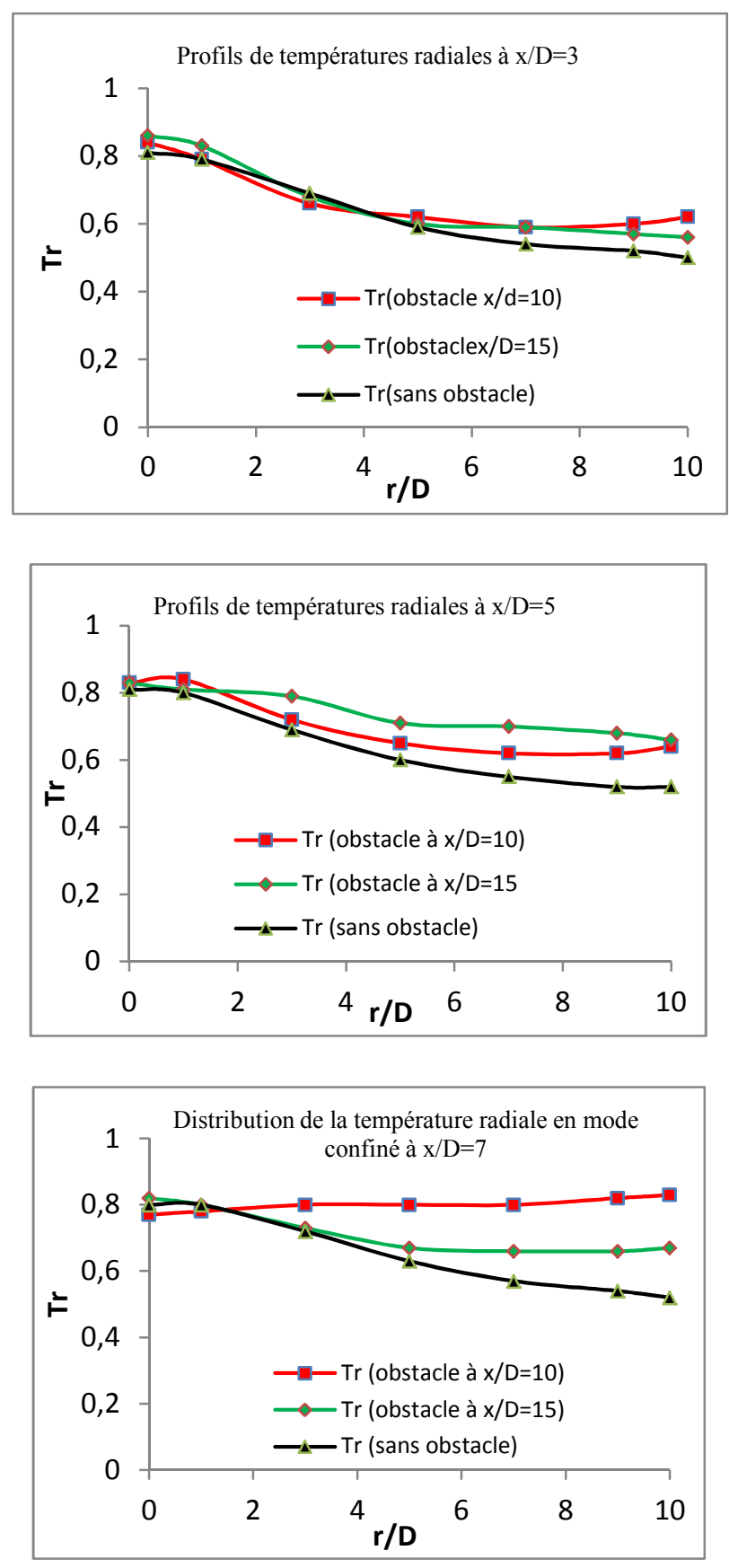

Figure 8. Comparaison des profils des températures axiales et radiales d'un multi jets tourbillonnaire en mode confiné avec et sans obstacle.

La présence de l'obstacle a pour conséquence un retour d'air chaud qui induit l'augmentation de l'amplitude thermique et une meilleure homogénéisation aux bords du jet résultant comme on le constate sur la figure (8). La distribution de la température radiale à la station axiale $\mathrm{x} / \mathrm{D}=7$ montre que l'obstacle placé à 10 diamètres de l'orifice de soufflage assure une meilleure homogénéité et stabilité thermique avec un étalement notable, en comparaison avec les deux autres configurations où l'obstacle est placé à 15 diamètres de l'orifice de soufflage et où l'écoulement est confiné sans obstacle.

Loin, à l'aval des diffuseurs, l'impact donne une nette homogénéisation thermique. Contrairement à l'évolution axiale, la décroissance radiale des maxima de températures est beaucoup moins accentuée pour le jet multiple que pour le jet simple.

\section{Conclusion}

Pour le jet tourbillonnaire impactant, l'obstacle fait accroître la turbulence davantage dans la partie centrale de l'écoulement confiné et influence peu la zone périphérique du jet jusqu'à une certaine distance appropriée du diffuseur.

La présence de l'obstacle dans le cas d'un multi jet tourbillonnaire en mode confiné a pour conséquence un retour d'air chaud qui induit l'augmentation de l'amplitude thermique et une meilleure homogénéisation aux bords du jet résultant et permet aussi un étalement appréciable. Aussi le confinement en présence de l'obstacle favorise d'une manière non négligeable la stabilité en température et d'autant plus lorsqu'on s'éloigne de l'orifice de soufflage.

\section{References}

1. M. Braikia, L. Loukarfi, A. Khelil, H. Naji, "Improvement of thermal homogenization using multiple swirling jets", THERMAL SCIENCE, 2012, Vol. 16, No. 1, pp. 239-250. 239.

2. Nakod, P.M., Prabhu, S.V., Vedula, R.P., "Heat transfer augmentation between impinging circular air jet and flat plate using finned surfaces and vortex generators", Experimental Thermal and Fluid Science, 32 (2008), pp.1168-1187.

3. Felli, M., Falchi M., Pereira, F.G.A., "Distance effect on the behaviour of an impinging swirling jet by PIV and flow visualizations", Experiments in Fluids, 48 (2010), pp. 197-209.

4. Braikia M., Loukarfi L., Djafer L., "Homogénéisation thermique par multijets rotationnels". CIM 2005, $12^{\mathrm{eme}}$ Congre International de Métrologie 20-24 Juin 2005, LYON-FRANCE.

5. Braikia M., Khelil A., Loukarfi L., Naji H. «parameters optimization of a multi-swirling jet diffuser » $X^{\mathrm{e}}$ Conférence sur la ventilation industrielle, du 17 au 19 septembre 2012, Paris, France.

6. Loukarfi L., Braikia M., Djafer L., "Amélioration de la qualité des mélanges par multijets rotationnels : résultats de synthèse" CFM 2003, 1-5 septembre 2003, Nice, France. 